\title{
Effect of Co-monomer Styrene on Reactive Compatibilization of PE/Wood-flour Composites
}

\author{
Yuewen $\mathrm{Li}^{1 *}$, and Xinhua Chen ${ }^{1}$ \\ ${ }^{1}$ Hunan Vocational College of Science \& Technology, 410004 Changsha, China
}

\begin{abstract}
Compatibilization between polyethylene(PE) and wood-flour was achieved by means of direct reactive extrusion of PE, wood-flour, maleic anhydride(MAH), co-monomer styrene(St) and dicumyl peroxide. Mechanical properties and heat distortion temperature(HDT) of the $\mathrm{PE} /$ wood-flour composites were tested, and impact cracking surfaces of the composites were observed via scanning electron microscope. Effect of St on reactive compatibilization of the composites was evaluated. Experimental results indicated that the anchoring strength of interface in the composites was obviously strengthened after St was added. Mechanical properties and HDT of the composites raised with increase of use level of St on the initial stage, and then gradually descended. When use level of St ran up to $1.5 \%$, mechanical properties and HDT of the composites were best. At this moment, the mole ratio of St and MAH was approximately 1:1.
\end{abstract}

\section{Introduction}

Wood/plastics composite(WPC) has usually poor compatibility, and compatibilization on WPC can notably raise its property. Dealing with wood-flour via reaction with silane ${ }^{[1]}$, titanate $^{[2]}$, acetic anhydride ${ }^{[3]}$ and benzoyl chloride ${ }^{[4]}$ can reduce polar disparity of wood and plastics, and improve compatibility between them. Macromolecular compatibilizers such as glycidyl methacrylate graft copolymer ${ }^{[5]}$, maleic anhydride graft copolymer ${ }^{[6]}$ and acrylic vinyl monomer copolymer ${ }^{[7]}$ not only contain epoxy group, anhydride and carboxyl which can react with hydroxyl on wood-flour, but also contain macromolecular chain which have favorable compatibility with resin, thus they have commendable compatibilization on WPC.

In the process of melt blending of plastic and wood-flour, direct adding MAH and initiator could improve compatibility of WPC. But percent grafting of MAH on macromolecular chain was low because of complexity of reaction environment, hindering further improvement of WPC property. In this research, it was tried increasing percent grafting of MAH via adding co-monomer styrene(St) to improve compatibilization effect in the process of reaction compatibilization of polyethylene (PE)/wood-flour composites, and influence regular pattern of St dosage on composite property was discussed.

\footnotetext{
*Corresponding author:lyw1965@126.com
} 


\section{The experiment}

\subsection{Materials}

PE, 2911, Wushun Subsidiary Company, Sinopec Group. Wood-flour, particle size $150 \mu \mathrm{m}$. MAH, A.R., Tianjin Kermel Chemical Reagent Co. Ltd.. St, A.R., Tianjin Damao Chemical Reagent Plant. Dicumyl peroxide(DCP), C.P., Shanghai Lingfeng Chemical Reagent Co. Ltd..

\subsection{Preparation method and process parameters}

Wood-flour was dried for 12 hour at $100{ }^{\circ} \mathrm{C}$ in advance. The raw materials including PE, wood-flour, MAH, St, and DCP were mixed together for $5 \mathrm{~min}$ in the SHR high-speed blender. The dosages of components were shown in Table 1 . The preliminary mixture was extruded at $180{ }^{\circ} \mathrm{C}$ via SHJ-20 co-direction twin-screw extruder, then cooled to room temperature and broken up to granules. The final standard test samples were prepared on the GEK80 injection moulding machine. The injection temperature was $180{ }^{\circ} \mathrm{C}$, and the injection pressure was $110 \mathrm{MPa}$.

Table 1. Composition of composite samples

\begin{tabular}{ccccccccc}
\hline Composite number & $1 \#$ & $2 \#$ & $3 \#$ & $4 \#$ & $5 \#$ & $6 \#$ & $7 \#$ & $8 \#$ \\
\hline PE(wt\%) & 70 & 68.74 & 68.39 & 68.04 & 67.69 & 67.34 & 66.99 & 66.64 \\
Wood-flour(wt\%) & 30 & 29.46 & 29.31 & 29.16 & 29.01 & 28.86 & 28.71 & 28.56 \\
MAH(wt\%) & 0 & 1.5 & 1.5 & 1.5 & 1.5 & 1.5 & 1.5 & 1.5 \\
DCP(wt\%) & 0 & 0.3 & 0.3 & 0.3 & 0.3 & 0.3 & 0.3 & 0.3 \\
St(wt\%) & 0 & 0 & 0.5 & 1.0 & 1.5 & 2.0 & 2.5 & 3.0 \\
\hline
\end{tabular}

\subsection{Morphology observation and properties testing}

Impact cracking surfaces of samples were observed with QUANTA-200 SEM after sputtercoated with platinum. HDT was tested according to ISO75-2:2003(A) on RV-300 thermal deformation Vicat softing point temperature tester. Tensile and flexuous tests were respectively performed according to GB/T 16421-1996 and GB/T 9341-2000 on WDW3020 micro-control electron universal test machine. Impact tests were performed according to GB/T 1043-1993 on XJC-25ZD electron impact tester.

\section{Results and discussions}

\subsection{Reactive compatibilization mechanism of the composite}

In melting extrusion process of PE, wood-flour, MAH and DCP, DCP decomposed to produce primary free radical, and it captured hydrogen atom on PE molecule chain, resulting of creation of PE macromolecule free radical, then it reacted with MAH to create MAH grafted PE(PE-g-MAH). PE long chain of PE-g-MAH could integrate into PE matrix, and MAH radical group could react with hydroxyl on wood-flour, thus the wood-flour and plastic were bonded together. In melting graft process of MAH with PE, percent grafting of MAH was usually low, and cograft of proper second monomer could effectively raise its percent grafting ${ }^{[8,9]}$. Co-monomer is usually electron rich monomer, such as St. St could more quickly react with PE macromolecule free radical than $\mathrm{MAH}$, and generated St radical 
could exist longer, and it could more rapidly react with MAH than PE macromolecule free radical, resulting of increase of percent grafting of MAH. In other research, it was considered that MAH could form electron transfer complex with $\left.\mathrm{St}^{[10}\right]$, then it could more rapidly be grafted onto $\mathrm{PE}$ macromolecule chain. Addition of St speeded up grafting reaction between macromolecule free radical and $\mathrm{MAH}$, and reduced cross-linking opportunity between macromolecule free radicals, and prevented degradation of processing properties of composites. Compatibilization reactions of MAH and St on the composites are shown in Fig.1.

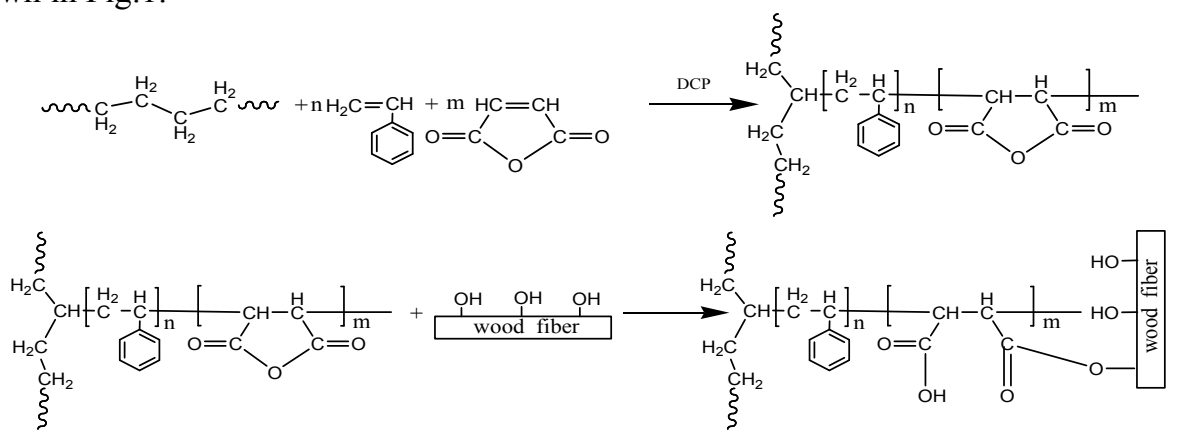

Fig. 1. Compatibilization reactions of MAH and St on the composites

\subsection{Morphology of impact cracking surfaces of the composites}

SEM micrographs of impact cracking surfaces of the composites are shown in Fig.2. Fig.2(a) is the SEM micrograph of impact cracking surface of 1\# WPC, which wasn' $t$ compatibilized. It can be observed that there is obvious gap between wood-flours and matrix, which indicates weak interfacial adhesion between PE and wood-flours in the noncompatibilized composites. Fig.2(b) and (c) are respectively the SEM micrographs of impact cracking surface of $2 \#$,and $5 \#$ composites. In melting extrusion process of $2 \#$ composites, MAH and DCP were added; it can be observed that combination of PE and wood-flours is compact. But peeling phenomenon between wood-flour and PE appeared under the action of impact force, which indicates that matrix stress didn't completely be transmitted to wood-flour. In melting extrusion process of 5\# composites, MAH, DCP and co-monomer St were added, it can be observed that interface between PE and wood-flour is obscure, and peeling phenomenon didn't appear under the action of impact force, which indicates that addition of St improved compatibilization effect, and heightened bonding strength of the composites.

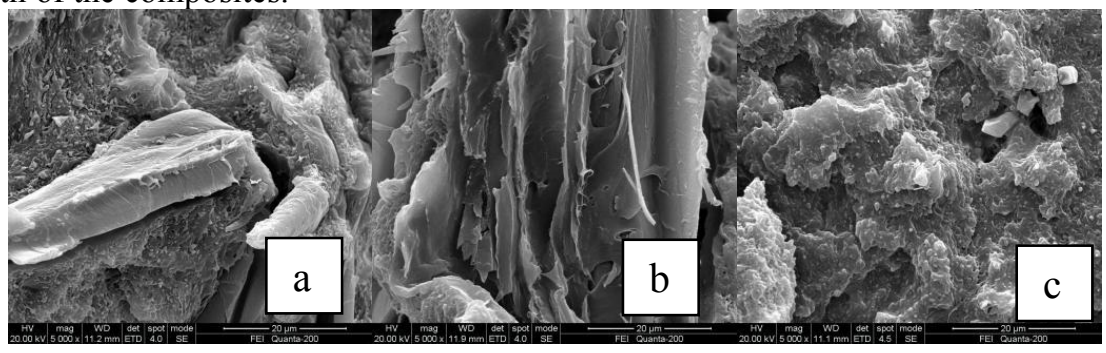

Fig. 2. SEM micrographs of impact cracking surfaces of the composite samples: (a)1\#, (b)2\#, (c)5\# 


\subsection{Effect of reactive compatibilization on mechanical property and HDT of the composites}

Mechanical property and HDT of $1 \#$ and $2 \#$ composites are shown in Table 2. 1\# composites didn' $t$ carry through compatibilization treatment, and $2 \#$ composites carried through reactive compatibilization via MAH and DCP. It can be found that tensile strength, flexural strength, notched impact strength, elongation at break and HDT increased obviously, which indicates that direct reactive compatibilization can effectively improve property of the composites. After direct reactive compatibilization, compatibility of the composites increased, and combination between PE and wood-flour become close together, therefore matrix stress could effectively be transmitted to wood-flour, resulting of increase of tensile strength, flexural strength, notched impact strength and elongation at break. When heated, matrix stress of the composites after compatibilization could be transmitted to wood-flour as before, and wood-flour resistance to thermal deformation excels obviously PE, thus HDT of the composites heightened evidently.

Table 2. Comparison of mechanical property and HDT of the composites before and after reactive compatibilization

\begin{tabular}{cccccc}
\hline WPC number & $\begin{array}{c}\text { Tensile } \\
\text { strength } \\
(\mathrm{MPa})\end{array}$ & $\begin{array}{c}\text { Flexural } \\
\text { strength(MPa) }\end{array}$ & $\begin{array}{c}\text { Elongation at } \\
\text { break }(\%)\end{array}$ & $\begin{array}{c}\text { Notched } \\
\text { impact } \\
\text { strength }(\mathrm{KJ} / \mathrm{m} \\
2\end{array}$ & HDT $\left({ }^{\circ} \mathrm{C}\right)$ \\
\hline $1 \#$ & 19.2 & 23.7 & 8.4 & 3.5 & 70.4 \\
$2 \#$ & 32.8 & 29.7 & 11.5 & 5.1 & 78.2 \\
\hline
\end{tabular}

\subsection{Effect of St dosage on mechanical property of the composites}

Influence law of St dosage on mechanical property of the composites is shown in Fig.3Fig.6. When St dosage was less, tensile strength, flexural strength, notched impact strength and elongation at break of the composites increased with the increase of St dosage. Mechanical properties of the composites were the best at $1.5 \%$ dosage of St. After that, with the increase of St dosage, mechanical properties of the composites decreased gradually. When MAH grafted with PE alone, its grafting points were less, and graft chain length was shorter, resulting of low grafting rate of MAH. After adding co-monomer St, MAH could be grafted onto PE molecular chain faster and more with St as mediator, and grafting rate increased, resulting that bonding strength of the composites increased, and the mechanical properties were also improved. When the St dosage reached $1.5 \%$, the molar ratio of St to MAH was about 1:1. This moment, whether St and MAH reacted with the macromolecular free radicals one after another, or St and MAH in the form of electron transfer complexes reacted with the free radical, St could give full play to the role of bridge between MAH and $\mathrm{PE}$, and the grafting rate of MAH was highest, and bonding strength of the composites was firmest, and the mechanical properties were also best. When the amount of St continued to increase, there were some excess St molecules. Because of the existence of a competitive relationship between excess St molecules, MAH and electron transfer complexes, the chance of MAH grafting with PE was reduced, resulting that the grafting rate of MAH and mechanical properties of composites decreased gradually. 


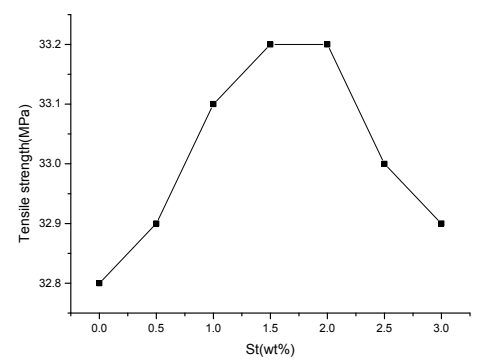

Fig. 3. Effect of St dosage on tensile strength

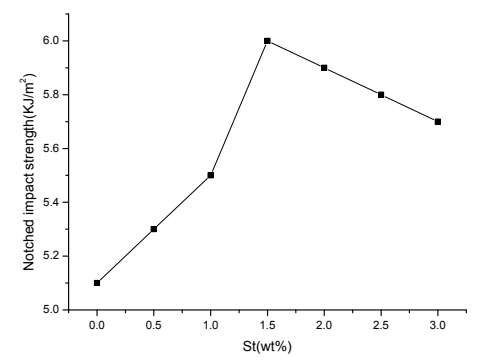

Fig. 5. Effect of St dosage on notched impact strength

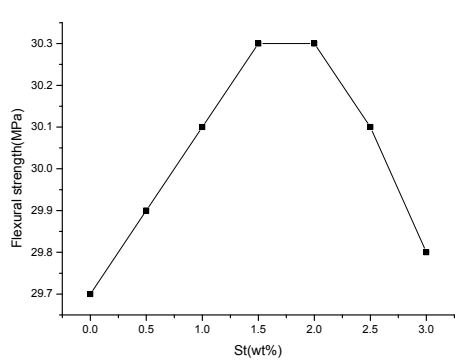

Fig. 4. Effect of St dosage on flexural strength

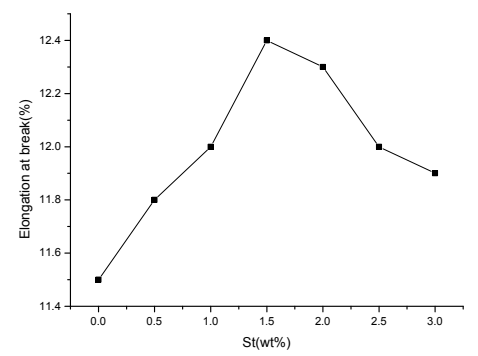

Fig. 6. Effect of St dosage on elongation at break

\subsection{Effect of St dosage on HDT of the composites}

Relationship between HDT of the composites and St dosage is shown in Fig.7. The variation of HDT with the St dosage was consistent with the mechanical properties of the composites. HDT of the composites reflected resistance to bending deformation under heating conditions. Resistance of PE matrix of the composites to bending deformation under heating conditions descended, but resistance of wood-flours was less affected by temperature. Grafting ratio of MAH was greater, combination of the composites was more compact, and stress born by PE matrix could be more transferred to wood-flours, and HDT of the composites was higher.

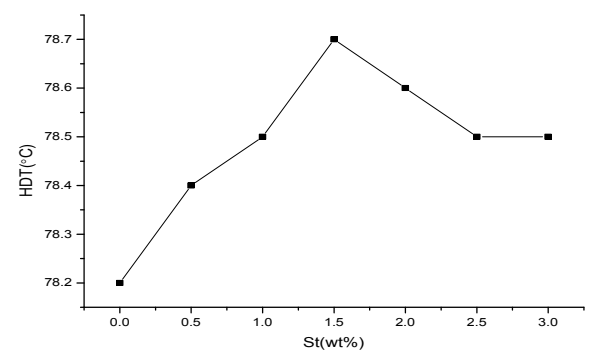

Fig. 7. Effect of St dosage on HDT of the composites

\section{Conclusions}

(1) SEM images showed that combination of the composites was more compact after adding the co-monomer St in the process of reactive compatibilization. 
(2) Mechanical properties and HDT of the composites were significantly improved after reactive compatibilization.

(3) In the process of reactive compatibilization, mechanical properties and HDT of the composites increased with the increase of St dosage at initial stage, then gradually decreased. When use level of St ran up to $1.5 \%$, mechanical properties and HDT of the composites were best. At this moment, the molar ratio of St to MAH was about 1:1.

\section{References}

1. M. B. N. Sonia, S. C. Graziela, M. L. R. Simone, Polymer Testing, 26, 619(2007)

2. L. L. Yang, H. Li, Z. Y. Zhong, Plastics Science and Technology, 38, 36(2010)

3. T. F. Qin, H. P. Yan, China Wood Industry, 13,17(1999)

4. R. C. Mankikadan, S. M. Nair, T. Sabu, Jorunal of Applied Polymer Science, 1483(1996)

5. C. Xiong, R. R. Qi, Engineering Plastics Application, 36, 22(2008)

6. T. Liu, S. R. Lu, Y. L. Wang, C. X. Zhang, Z. Y. Huang, Plastics Science and Technology, 38, 58(2010)

7. R. Y. Wang, C. Y. Wan, Y. Zhang, Y. X. Zhang, China Plastics, 19, 63(2005)

8. X. M. Xie, N. H. Chen, S. Li, Acta Polymerica Sinica, 351(1999)

9. B. D. Zhu, J. Wang, H. L. Li, W. C. Dong, C. Dong, China Plastics, 26, 50(2012)

10. Y. Li, X. M. Xie, B. H. Guo, Polymer, 42, 3419(2001) 